First publ. in: Journal of Low Temperature Physics 137 (2004), 3-4, pp. 335-344

\title{
Electrons above helium films on metal substrates
}

\author{
J. Angrik, A. Faustein, J. Klier, and P. Leiderer \\ Department of Physics, University of Konstanz, D-78457 Konstanz, Germany
}

\begin{abstract}
We report about our investigations on two-dimensional electron ensembles floating above thin helium films supported by metallic substrates. We found a surprisingly high stability of these electrons, although the substrates were conducting which allows breakthrough at weak spots of the helium film due to roughness peaks. We have measured the electron densities and corresponding relaxation times. By pulling the electrons from the area above the loading electrode to the area above a separate electrode one can enhance the lifetime of these surface state electrons.
\end{abstract}

PACS numbers: 67.70.+n, 73.90.+f, 73.50.-h

\section{INTRODUCTION}

The system of electrons on liquid helium, which is under investigation now already for more than three decades, has attracted much attention due to some of its very unique features, see e.g. [1]. One of them is its nearly ideal two-dimensionality (2D) in a very clean surrounding with well defined interactions [2], namely the Coulomb interaction, gas atom scattering, and ripplon (quantized surface wave) scattering. Unlike in semiconductors, were similar two-dimensional electron systems have been studied extensively, the electron density is generally significantly lower and can easily be varied over at least four orders of magnitude. One of the most interesting phenomena is the Wigner crystal. By increasing the electron density one can study the phase transition from a classical dilute electron gas into the Wigner crystal $[3,4]$. While this phase transition is experimentally investigated and well understood, the second phase transition occurring at higher electron densities, when the Wigner crystal melts into a degenerated Fermi gas (quantum melting), eludes itself from detailed observation up till now. The surface of bulk helium becomes unstable due to an electrohydrodynamical instability when the electron density exceeds $2.4 \times 10^{13} \mathrm{~m}^{-2}$ [5]. Higher electron

Konstanzer Online-Publikations-System (KOPS)

URL: http://www.ub.uni-konstanz.de/kops/volltexte/2007/2732/

URN: http://nbn-resolving.de/urn:nbn:de:bsz:352-opus-27323 


\section{J. Angrik, A. Faustein, J. Klier, P. Leiderer}

densities $\left(2 \times 10^{16} \mathrm{~m}^{-2},[6]\right)$ can be reached above thin helium films, which are additionally stabilized by van der Waals attraction to the substrate [7]. Therefore they are in principle suitable to bridge the gap between the classical and quantum mechanical regime. However the underlying substrate surface, its roughness and surface charges, pose new problems and make the system experimentally challenging.

In order to investigate the system of electrons on helium films we have used optical methods, by which we determine the helium film thickness on our substrates. Since the helium film gets depressed by the electrostatic pressure of the electrons floating on it, the measurement of the actual and initial film thickness allows a direct determination of the electron density using the known relation [8]

$$
d=d_{0}\left(1+\frac{n_{e}^{2} e^{2}}{2 \epsilon_{0} \rho g H}\right)^{-\frac{1}{3}},
$$

where $d$ is the thickness of the charged film, $d_{0}$ the uncharged (initial) film thickness, $n_{e}$ the electron density, $\rho$ the density of the liquid helium, $g$ the acceleration due to gravity, and $H$ the height of the sample above the bulk helium level. The measurement of the electron current and, simultaneously, the electron density should give a direct access to the mobility of electrons on such films.

\section{EXPERIMENTAL SETUP}

We use a double surface plasmon spectroscopy setup [9] (see Fig. 1) to measure simultaneously the helium film thickness at two different positions on the sample. The electrode arrangement consists of a structured thermally evaporated $45 \mathrm{~nm}$ gold film with isolated electric contacts on a BK7-glass prism. The sample surface is mounted vertically in the experimental cell of a ${ }^{3} \mathrm{He}$ cryostat, which allows optical access through glass windows, symmetrically placed in a 4 -fold geometry. A p-polarized light beam from a laser diode, split up by a beamsplitter, is resonantly coupled into surface plasmons at a certain angle of incidence and focused onto photodiodes outside the cryostat. Helium films adsorbed on the gold substrate cause a resonance detuning, and measurement of the shift of the resonance angle allows to determine the thickness of the films with a resolution of about $1 \AA$. The measured thickness is an average value given by the area of the laser spot, which is about $1 \mathrm{~mm}^{2}$.

The experiments were conducted in the temperature range 1.0 to $1.7 \mathrm{~K}$. At the beginning of each experiment adsorption isotherms were performed 
Electrons above helium films on metal substrates

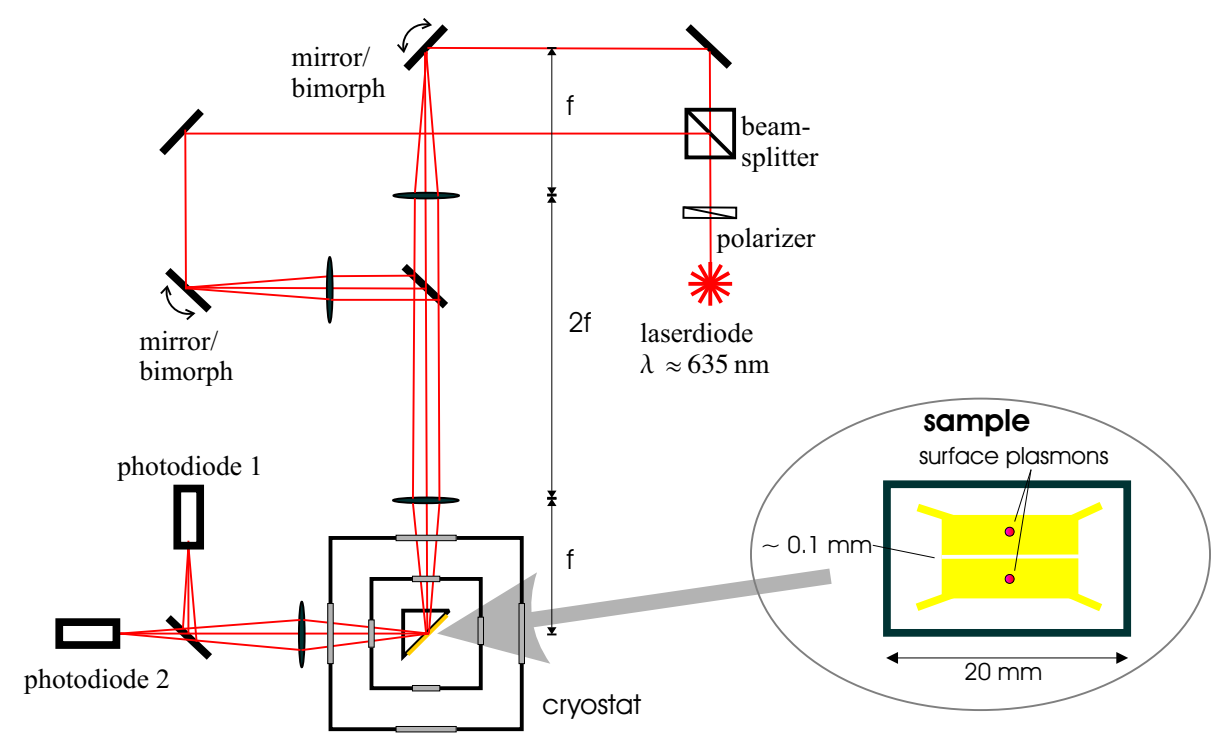

Fig. 1. Double surface plasmon spectroscopy setup. At a certain angle of incidence the two beams of p-polarized light can resonantly couple to surface plasmons on the sample, consisting of gold films evaporated onto a glass prism inside an optical cryostat. The inset on the right side shows a sketch of the structured sample, the typical position of the plasmons is indicated.

(by slowly condensing in ${ }^{4} \mathrm{He}$ into the experimental cell) to check the function of the spectroscopy setup. To measure at different initial helium film thicknesses the bulk level was varied by adding additional helium into the cell. Between the sample electrodes and an electrode in front of the sample an electric field can be applied, which acts as a holding field and guides the electrons to the sample surfaces. A small tungsten filament serves as the source for the electrons. Electrometers connected to the gold films measure the breakthrough currents at the different electrodes.

The substrates in our first experiments contained a guard ring, i.e., an electrode around the substrate that was kept on a slightly negative potential to prevent the electrons from flowing off laterally. However, it turned out that this guard ring had little influence on the measured electron densities and stabilities, so it was omitted and the substrate was structured into two segments separated by a gap of around $0.1 \mathrm{~mm}$ (see inset of Fig. 1). We used a CCD camera to watch the sample through one of the windows while adjusting the laser beams and positions of the surface plasmons (and hence the positions, at which the film thicknesses were measured). In addition a strong IR-laser $(\approx 500 \mathrm{~mW})$ was used, whose focus could be moved over the whole sample surface, to locally burn away the helium film and so discharge 


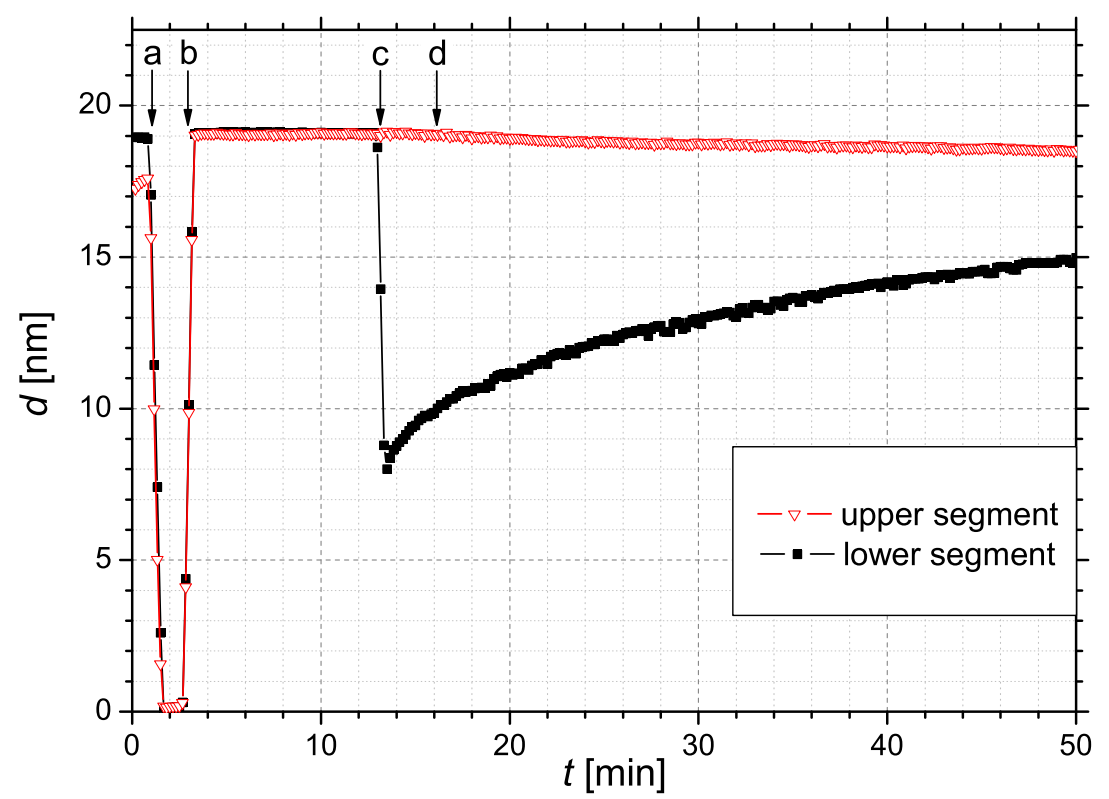

Fig. 2. Shown are the helium film thicknesses, $d$, measured at two different positions of the sample, one on the upper and one on the lower segment at $1.7 \mathrm{~K}$. The typical experimental procedure is as following: first the film is burned away (a) and thereby discharged. Then (b) we allow the system to settle for several minutes and check the initial film thickness, before the film is charged by pulsing the filament for some seconds (c). A positive voltage of $+15 \mathrm{~V}$ is applied to the lower segment, while the upper is kept at a negative potential of $-2 \mathrm{~V}$. About two minutes after switching off the filament $(\mathrm{d})$ a voltage of $-20 \mathrm{~V}$ is applied to the lower segment and $+20 \mathrm{~V}$ to the upper segment, to pull the electrons from the charged lower segment to the uncharged upper one.

the surface.

\section{MEASUREMENTS}

We measure the change in helium film thickness to determine the electron density on the film. A typical experimental procedure is shown in Fig. 2. In order to get reproducible conditions for every experiment, we usually burn away the helium film at the start of each measurement run, either by using an IR laser beam or by continuously firing the filament at high power. Thus we have a reliable and reproducible method to check the film thickness and, 


\section{Electrons above helium films on metal substrates}

by applying an additional negative electric potential of about $-100 \mathrm{~V}$ to the substrate electrodes, we can be sure to get rid of the electrons on top of the helium film. After the film reaches its initial saturation thickness, $d_{0}$, we allow the system to settle for several minutes. As one clearly observes (Fig. 2, b) the film thickness is stable during this time.

By pulsing the filament, usually with $5 \mathrm{~Hz}$ and pulse length $30 \mathrm{~ms}$, and applying a positive electric holding field of a few Volts $/ \mathrm{cm}$, it is possible to charge the helium film, and the corresponding depression of the film thickness is measured. After the filament is turned off we observe in general a relaxation of the film thickness towards its initial (uncharged) value, indicating a loss of electrons from the surface.

Using the two surface plasmon spectroscopy setups in parallel allows, on one hand, to check the measured signals against each other, and, on the other hand, to perform transport measurements. The latter can be achieved by charging the film above only one of the two separately contacted substrate segments. A negative voltage applied to the other segment prevents the helium film above it to become charged. After switching off the filament we apply a electric field between the two segments, and so pull some of the electrons from the charged helium film to the initially uncharged film above the other segment over the insulating gap (see inset of Fig. 1).

\section{RESULTS}

We were able to create high electron densities of up to $10^{15} \mathrm{~m}^{-2}$ above our metal substrates [10]. The stability of these electrons can be very high, but depends very much on the used substrate. The reproducibility of these measurements is good for each of the substrates, but comparison between different ones is not directly possible due to differences in stability behavior and maximum electron density. The relaxation times differ from substrate to substrate. Times between a few minutes up to many hours $(>15 \mathrm{~h})$ where measured.

A typical relaxation curve is shown in Fig. 3. The first minutes after turning off the filament show the fastest losses of electrons, which can be described by an exponential decay law. Afterwards, we usually observe a transition into a linear decay regime, where loss rates are constant over long times (from a few minutes up to many hours, depending on the substrate). Measurements at different temperatures, from $1.0 \mathrm{~K}$ up to $1.7 \mathrm{~K}$, show no significant temperature dependence of this loss mechanism, although the scattering of the electrons due to helium vapor atoms is far less at low temperatures. It is likely therefore that the substrate roughness is the crucial 
J. Angrik, A. Faustein, J. Klier, P. Leiderer

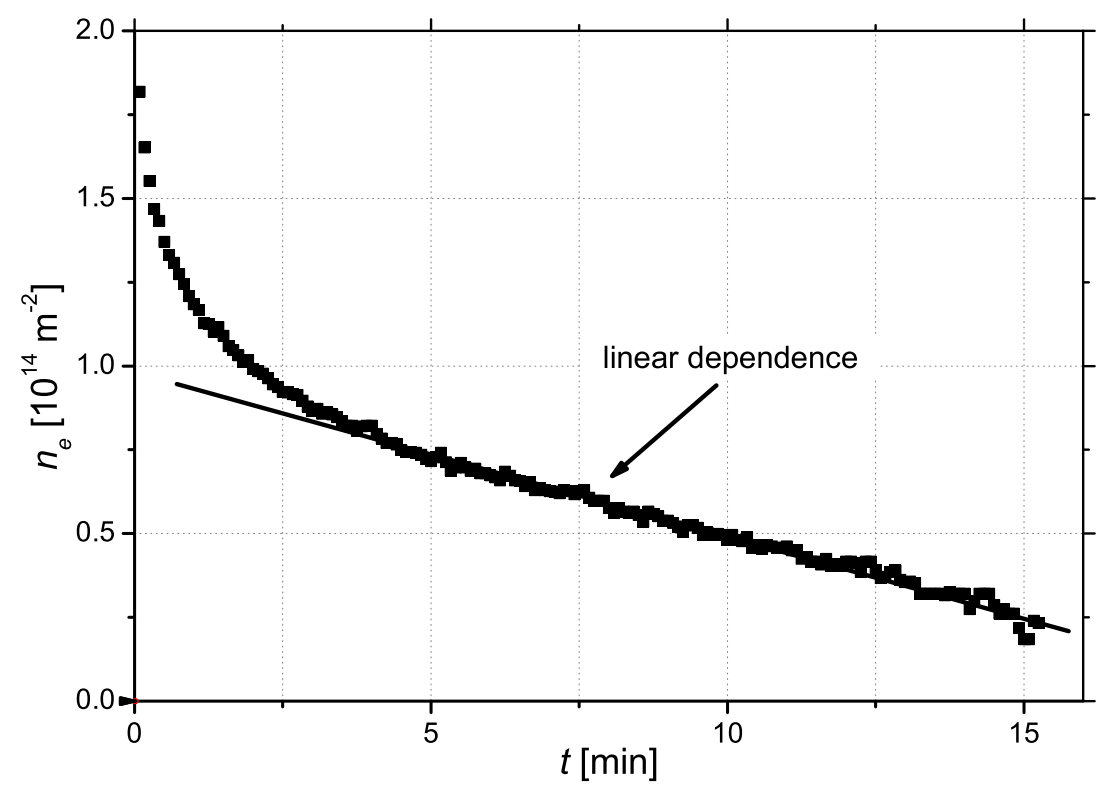

Fig. 3. Typical relaxation curve at $1.7 \mathrm{~K}$ with an exponential decay of electrons at the beginning that passes into a linear decay regime. The maximum electron density is moderate and the relaxation time is short on this substrate.

parameter for this loss mechanism. The possibility that electrons did escape into the vapour space can be excluded. After charging the surface even strong repulsive potentials (up to $-50 \mathrm{~V} / \mathrm{cm}$ ) did not show any influence on the decay-rate of the electrons.

A transport measurement of surface state electrons (SSE) from the upper segment to the lower over the insulating gap is presented in Fig. 4. In contrast to the measurement shown in Fig. 2 this time the helium film above the upper segment was charged. An electron density of $10^{15} \mathrm{~m}^{-2}$ on the upper segment was achieved (not shown) and at time $t=0$ (mark "a" in Fig. 4) a potential difference of $120 \mathrm{~V}$ [11] between the two segments was applied. As the signal shows, an electron transport is observed indeed: the electron density on the lower segment increases, at first fast, then slower as the electron density on the upper segment drops. At (c) a maximum electron density of $1.9 \times 10^{14} \mathrm{~m}^{-2}$ above the lower segment is achieved. We believe that this is not the saturation density for this substrate, but depends on the waning electron reservoir at the upper substrate. After this reservoir is completely emptied (d) a linear decay behavior is observed that lasts for over $14 \mathrm{~h}$, only interrupted by a new measuring sequence. The slope of the 
Electrons above helium films on metal substrates

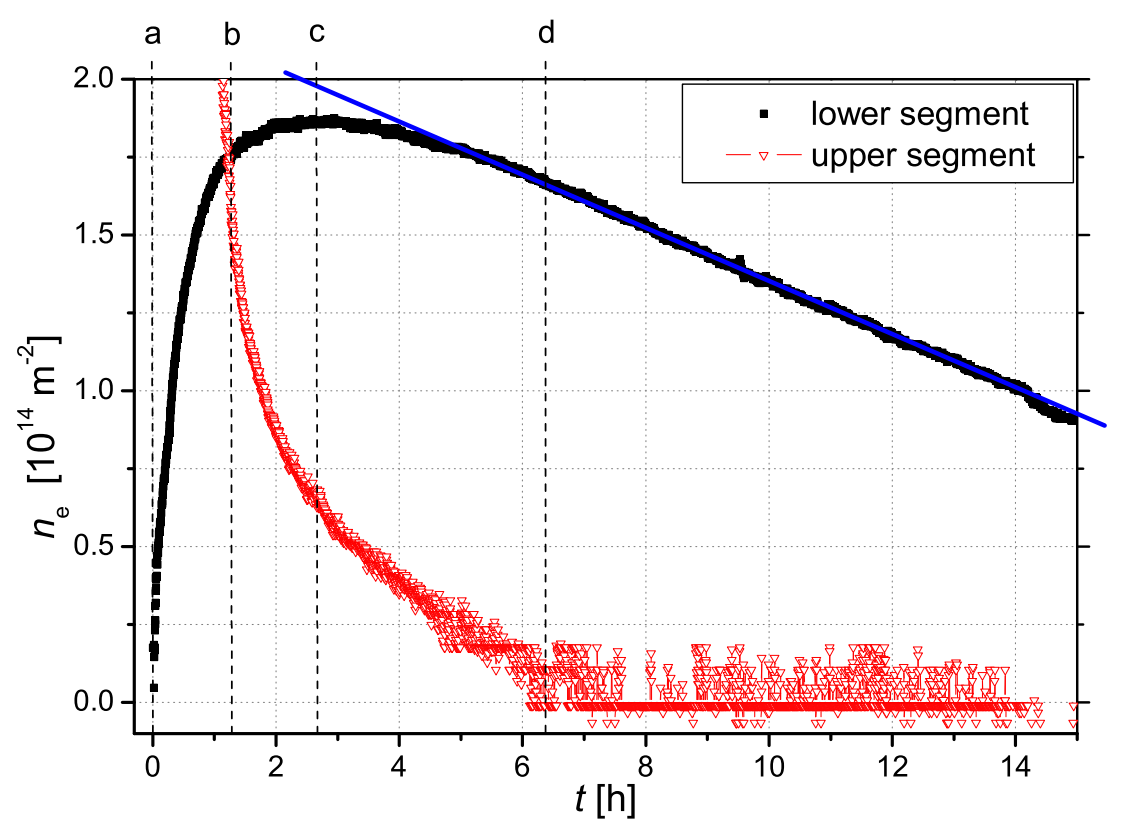

Fig. 4. Electron densities on upper and lower segments during a transport measurement at $1.7 \mathrm{~K}$. Here only the upper segment was charged by pulsing the filament. After switching off the filament a drain voltage of $-60 \mathrm{~V}$ at the upper and $+60 \mathrm{~V}$ at the lower segment is applied (a). At (b) the electron density on the lower segment increases by about 7000 electrons $\mathrm{s}^{-1}$, while the loss on the upper segment is nearly one order of magnitude higher. At (c) the maximum density on the lower segment is reached while the loss on the upper segment totals about 11000 electrons $\mathrm{s}^{-1}$. At (d) the upper segment is discharged and therefore the electron transport stops. The electron density on the lower segment now shows a linear decay of only about 5000 electrons $\mathrm{s}^{-1}$. So the stability of electrons on this lateral loaded film is extremely high.

curve corresponds to an electron loss of about $5000 \mathrm{~s}^{-1}$ from the substrate $(1.2 \mathrm{~cm} \times 0.4 \mathrm{~cm})$, which is equal to a current of $0.8 \mathrm{fA}$. If we assume this loss to be constant in time (which appears plausible) the electron current over the insulating gap totals to $1.9 \mathrm{fA}$ at Fig. 4(b) and $0.8 \mathrm{fA}$ at (c).

The electron loss from the helium film (shown in Fig. 5) as well as the observed transport showed no significant temperature dependence in the investigated range from $1.0 \mathrm{~K}$ up to $1.7 \mathrm{~K}$. We therefore believe this is not a thermally activated process. The details of the loss mechanism still have to be studied. 


\section{J. Angrik, A. Faustein, J. Klier, P. Leiderer}

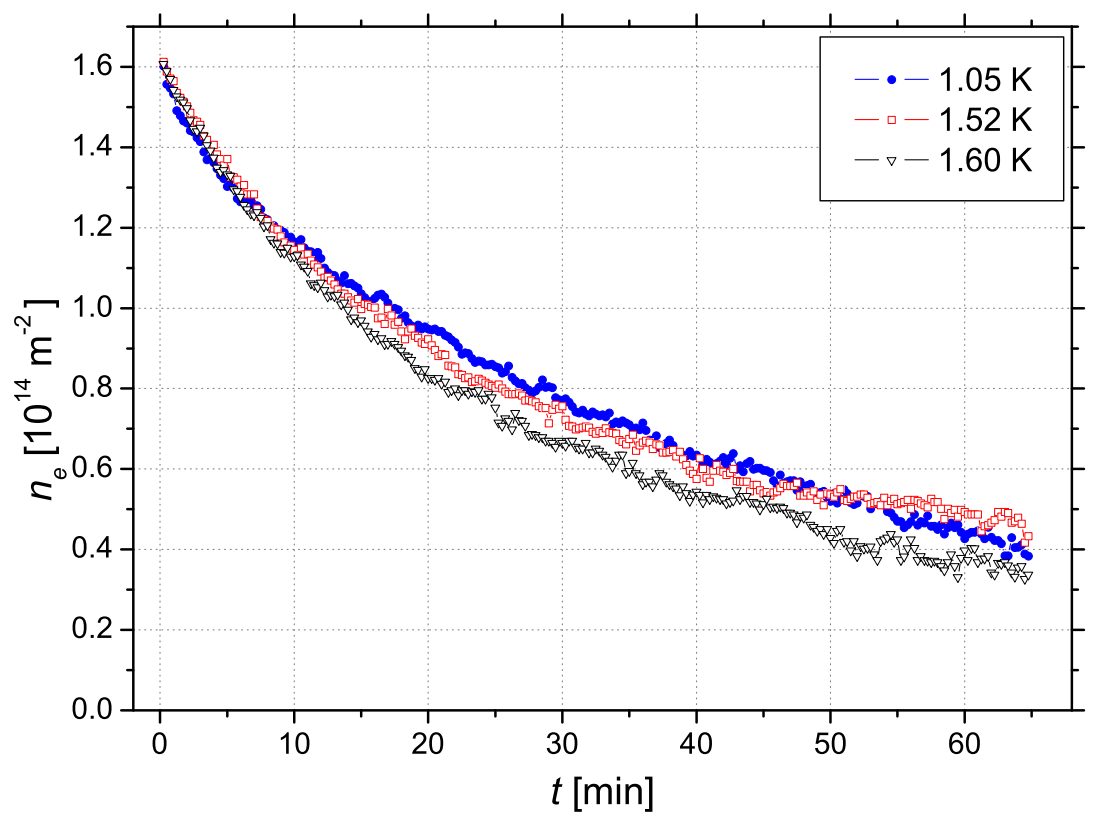

Fig. 5. A comparison of the stability of electrons at different temperatures on the same substrate. The films were charged at $1.7 \mathrm{~K}$, then the cryostat was immediately cooled down to the given temperature. No significant temperature dependence is discernible.

\section{DISCUSSION AND OUTLOOK}

Our measurements show for the first time that SSE above thin helium films ( $\gtrsim 100 \AA$ ) on metal substrates can be stable for many hours. Nevertheless we always observed a decay: the electrons flow off through the helium film into the substrate. Their stability as well as lateral transport properties are extremely surface dependent, some substrates showed very good stability and transport abilities, on others transport is hardly possible. We believe that pinned electrons [12] near the isolating gap play a dominant role for the transport properties, sometimes preventing electrons from lateral transport due to their electrostatic repulsion.

Another possible explanation for the lateral stability of the electrons, as well as a not discernible temperature dependence of the transport over the insulating gap can be found in Fig. 6. There the potential for SSE on $200 \AA$ helium films on the used sample (consisting of the image potential and the applied potential difference between the two electrode segments) is shown. The high potential barrier of $24 \mathrm{meV}$ makes a tunneling process 


\section{Electrons above helium films on metal substrates}

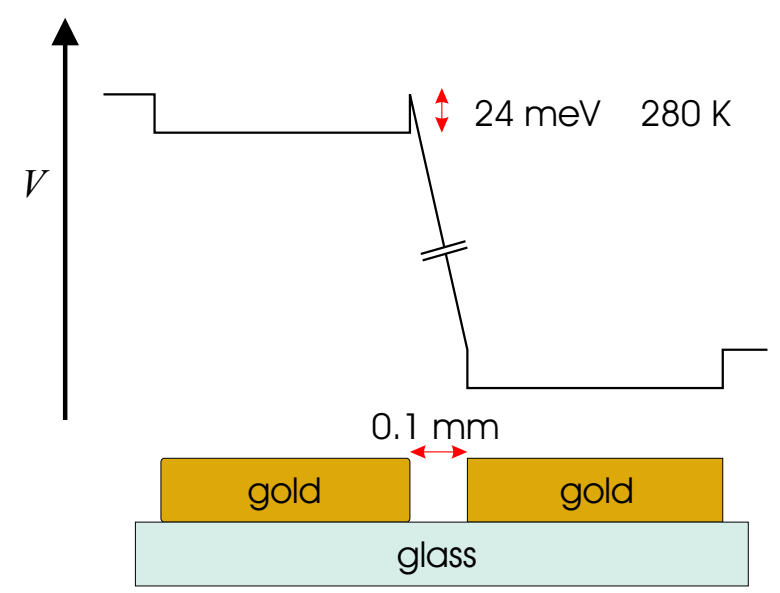

Fig. 6. Sketch of the potential $V$ (not to scale) for electrons above a $200 \AA$ helium film on the metal/glass substrate. The stronger image potential of electrons above the metal substrate compared to the glass substrate leads to a potential barrier of $24 \mathrm{meV}$. Shown is the situation for an applied potential difference between the two electrode segments of our sample.

likely. This also explains why in our setup the influence of a guard ring could be neglected, as the distance between substrate and guard ring was approximately $2 \mathrm{~mm}$.

In our measurements so far the history of the system was crucial, since the loss characteristic depended very much on the process of how the helium film was charged. Electrons pulled laterally onto the helium film above a metal substrate showed a much higher stability compared to filamentcharged helium films. If it is possible to recharge the latter film without disturbing the electrons on the first one, even higher densities should be within reach.

\section{ACKNOWLEDGMENTS}

This work was supported by the EU-RTN "Surface electrons on mesoscopic structures" and DFG-Grant Kl 1186/2. Experimental contributions by M. Köppl are gratefully appreciated.

\section{REFERENCES}

1. E.Y. Andrei, ed., Two-dimensional electron systems on helium and other cryogenic substrates, Kluwer academic publishers (1997). 


\section{J. Angrik, A. Faustein, J. Klier, P. Leiderer}

2. C.C. Grimes, T.R. Brown, Phys. Rev. Lett. 32, 280 (1974).

3. F.M. Peeters and P. M. Platzman, Phys. Rev. Lett. 50, 2021 (1983).

4. The variation of the $2 \mathrm{D}$ electron density and the resulting phase state is also interesting for many other system, e.g., for the 2D metal-dielectric transition [S.V. Kravchenko and M.P. Sarachik, Rep. Prog. Phys. 67, 1 (2004)] or even chemical and biological systems [A.Yu. Grosberg, T.T. Nguyen, and B.I. Shklovskii, Rev. Mod. Phys. 74, 329 (2002)].

5. M. Wanner, P. Leiderer, Phys. Rev. Lett. 42, 315 (1979).

6. X. L. Hu and A. J. Dahm, Phys. Rev. B 42, 2010 (1990).

7. H. Ikezi and P. M. Platzman, Phys. Rev. B 23, 1145 (1981).

8. H. Etz, W. Gombert, W. Idstein, and P. Leiderer, Phys. Rev. Lett. 53, 2567 (1984).

9. E. Kretschmann, Opt. Commun. 10, 353 (1974).

10. The density is well below the one for a Wigner crystal, which should occur at $\approx 2.5 \times 10^{15} \mathrm{~m}^{-2}$ for $T=1.7 \mathrm{~K}$.

11. We did not observe electron transport for voltage differences below $80 \mathrm{~V}$, equivalent to a field of $8 \mathrm{kV} / \mathrm{cm}$.

12. V. Shikin, J. Klier, I. Doicescu, A. Würl, and P. Leiderer, Phys. Rev. B. 64, 073401 (2001). 